\title{
Психофизиологические корреляты решения вербальных задач в ситуации смысловой инициации
}

\author{
Павел Н. Ермаков ${ }^{1}$, Екатерина Г. Денисова2" \\ ${ }^{1}$ Южный федеральный университет, г. Ростов-на-Дону, Российская Федерация \\ 2 Донской государственный технический университет, г. Ростов-на-Дону, Российская \\ Федерация \\ *E-mail: keithdenisova@gmail.com
}

\begin{abstract}
Аннотация
Ввеление. В статье представлены результаты исследования психофризиологических коррелят процесса решения вербальных задач на смысл. Новизна исследования заключается в использовании залач на смысл как инструмента моделирования процесса смыслообразования в условиях психофизиологического эксперимента. Авторы преАполагают, что характеристики электрической активности мозга могут отражать особенности процесса решения залач, направленных на раскрытие Аичностного смысла.
\end{abstract}

Методы. С целью проверки вылвинутой гипотезы использовался метоА электроэнцесралографрии (ЭЭГ). Регистрация электроэнцефралограммы мозга проводи^ась в 128 отведениях с использованием многоканального электроэнцедралографа «Нейровизор-136» (произволства компании «МКС», Россия). В исслеАОвании приняли участие юноши и Аевушки в возрасте от 18 Ао 25 ^ет, объем выборки состави^ 52 человека. Участникам эксперимента в интерактивном режиме предьявля^ись вербальные залачи, моделирующие ситуацию смысловой инициации, и эмоционально нейтральные логические залания.

Результаты. Установлено, что частотно-пространственные характеристики биоэлектрической активности коры головного мозга во время решения вербальных задач в ситуации смысловой инициации Аостоверно отличаются от показателей активности во время решения простых мыслительных залач $(p<0,05)$. Кроме того, отмечаются половые различия частотно-пространственных характеристик ЭЭГ $(\mathrm{p}<0,05)$.

ОбсужАение результатов. Повышение фрункциональной активности фрронтальных и центральных областей мозга, а также увеличение высокочастотной ритмики в темпорально-париетально-окципитальной зоне левого полушария могут отражать увеличение когнитивно-афрфективной нагрузки во решения залач в ситуации смысловой инициации.

Процесс смыслообразования, по-видимому, реализуется посреАством мультимоАального синтеза, при участии семантической и образной Аолговременной памяти. 


\section{КАючевые слова}

ЭЭГ-корреляты, энцефалография, психофизиология, смыслообразование, смыс^овая инициация, вербальные залачи, задачи на смысл, мозговые механизмы, мышление, мичностный смысл

\section{Основные положения}

- процесс решения вербальных залач в ситуации смысловой инициации отражает особенности процесса смыслообразования и характеризуется особой частотно-пространственной организацией биоэлектрической активности коры головного мозга;

- решение залач на смысл сопровождается снижением меАленноволновой активности Аельта- и тета- Аиапазонов, Аесинхронизацией альфра-ритма, а также увеличением относительного показателя представленности бета2- и гамма-ритмов; в полгруппах юношей и девушек наблюдаются различные изменения суммарной электрической активности коры головного мозга при сравнении показателей простой мыслительной Аеятельности и активности во время решения вербальных заАач в ситуации смысловой инициации;

- увеличение активности фрронтальных отАелов коры и выраженная активация левой темпорально-париетально-окципитальной зоны при решении вербальных задач в ситуации смысловой инициации характерны Аля подгруппы девушек;

- в полгруппе юношей изменения суммарной электрической активности коры головного мозга при решении вербальных залач на смысл менее выражены и в основном характеризуются повышением уровня активности фрронтальной коры и париетально-окципитальной области левого полушария.

\section{Для цитирования}

Ермаков П. Н., Денисова Е. Г. Психофизиологические корреляты решения вербальных задач в ситуации смысловой инициации // Российский психологический журнал. 2019. T. 16, № 1. С. 103-127. DOI: 10.21702/rpj.2019.1.5

Материалы статьи получены 08.08.2018 


\title{
Psychophysiological correlates of the performance of verbal tasks in situations initiating personal meanings
}

\author{
Pavel N. Ermakov ${ }^{1}$, Ekaterina G. Denisova ${ }^{2 *}$ \\ ${ }^{1}$ Southern Federal University, Rostov-on-Don, Russian Federation \\ ${ }^{2}$ Don State Technical University, Rostov-on-Don, Russian Federation \\ *Corresponding author. E-mail: keithdenisova@gmail.com
}

\begin{abstract}
Introduction. This paper presents the study of psychophysiological correlates of the performance of verbal tasks in situations initiating personal meanings. For the first time in psychophysiological research the authors employed meaning tasks as a tool for modeling the meaning-building process. The authors assume that certain patterns of the electrical activity of the brain may be associated with the process of solving verbal tasks in situations initiating personal meanings.
\end{abstract}

Methods. The study sample comprised 52 individual participants - boys and girls aged 18 to 25 years. The participants were visually presented with two types of tasks, namely (a) emotionally neutral logical tasks (control) and (b) verbal tasks that modeled situations of personal meaning initiation (test). The EEG activity was recorded from 128 leads using a multi-channel electroencephalograph (Neurovisor-136, Medical Computer Systems, LTD, Russia).

Results. Brain activity patterns during the solving of verbal tasks in a situation of personal meaning initiation significantly differ from activity during the solving of simple mental tasks $(p<0.05)$. The results indicate an increase in the functional activity of the frontal and central regions of the brain, as well as the temporal-parietal-occipital area of the left hemisphere. In addition to these differences, the results indicate that there were sex differences in the EEG frequency and spatial characteristics ( $p<0.05)$. Discussion. The increase in functional activity in frontal and central brain regions and the increase in high-frequency rhythms in the temporal-parietal-occipital area of the left hemisphere may be associated with an increase in cognitive and affective loads during the performance of verbal tasks in situations initiating personal meanings. It seems that creation of personal meaning is realized by means of multimodal synthesis and is associated with verbal and non-verbal long-term memory.

\section{Keywords}

EEG correlates, encephalography, psychophysiology, creation of personal meaning, meaning initiation, verbal tasks, meaning tasks, brain mechanisms, thinking, personal meaning 


\section{Highlights}

- The performance of verbal tasks in situations initiating personal meanings is associated with a certain frequency and spatial organization of the bioelectric activity of the cerebral cortex and characteristics of the process of personal meanings creation.

- The performance of 'meaning tasks' is accompanied by a decrease in the slowwave activity of delta and theta frequency bands, an increase in a relative index of beta2 and gamma rhythms, and alpha desynchronization.

- The comparison of psychophysiological correlates of mental activity during the performance of simple mental tasks and verbal tasks in situations initiating personal meanings demonstrated different changes in the electrical activity of the cerebral cortex in the subgroups of boys and girls.

- In the subgroup of girls, the performance of verbal tasks in situations initiating personal meanings is associated with the increased activity in the frontal cortex and activation in the left temporal-parietal-occipital area.

- In the subgroup of boys, changes in the electrical activity of the cerebral cortex during the performance of 'meaning tasks' are less evident and are mainly characterized by the increased activity in the frontal cortex and the parietal-occipital area of the left hemisphere.

\section{For citation}

Ermakov P. N., Denisova E. G. Psychophysiological correlates of the performance of verbal tasks in situations initiating personal meanings. Rossiiskii psikhologicheskii zhurnal - Russian Psychological Journal, 2019, V. 16, no. 1, pp. 103-127 (in Russian). DOI: 10.21702/rpj.2019.1.5

Original manuscript received 08.08.2018

\section{Введение}

Смысловая реальность как предмет научного исследования в психологии широко представлена в трудах отечественных ученых $[1,2,3,4,5,6]$. Исследователи обращают внимание на необходимость исследования механизмов смыслообразования [7, 8, 9]. В отношении стратегий смыслообразования в отечественной литературе нет единой позиции. Встречаются описания механизмов и стратегий усвоения смысла, классификации и стратегии образования личностных смыслов, а также пути формирования и способы трансляции смысловых установок [10, 11 и др.].

Исследование механизмов смыслообразования входит в проблематику когнитивной науки. Фундаментальные исследования мышления показали необходимость изучения не только движущих мотивов мысли, потребностей и интересов, побуждений и тенденций, которые детерминируют содержание и направленность мышления субъекта [12]. В свою очередь, изучение 
процессуальной стороны мыслительной деятельности уже давно вышло за рамки психологии и обратилось к методам психофизиологического исследования. С позиций смысловой теории мышления О. К. Тихомирова именно смыслообразование выступает как основной процесс интеграции новообразований в мышлении [13]. Ситуация смысловой инициации рассматривается как процесс объединения в акте мышления его когнитивного и аффективного компонентов, в результате которого появляется субъективно новое - личностный смысл. При этом интегративная роль смысловой сферы реализуется через опосредование порождения и функционирования когнитивных образований (образов, понятий, знаний) и процессов целеполагания $[2,9]$.

Психофизиологические особенности активности мозга в процессе мыслительной деятельности (решения вербальных задач) рассматриваются в ряде работ $[14,15,16,17,18,19,20$ и др.]. Активно исследуются мозговые механизмы решения креативных задач [21, 22, 23, 24 и др.]. В работах последних десятилетий изучаются нейрофизиологические корреляты стратегий и индивидуальных особенностей когнитивных процессов $[25,26,27,28]$, понятийного мышления [29], вербального интеллекта [30] и творческого мышления [31, $32,33,34]$. В рамках развития данной проблематики при помощи методов нейровизуализации была выстроена своя схема исследования, методология моделирования мыслительной деятельности в условиях психофизиологического эксперимента, накоплено огромное количество данных. Однако используемые в лабораторных условиях для моделирования мыслительного процесса задачи не дают возможность в полной мере изучить особенности обработки информации, связанной с индивидуальным опытом и смысловой сферой личности, а работ, посвященных психофизиологическим коррелятам данного процесса, на сегодняшний день практически нет.

В этой связи актуальным становится комплексное изучение процесса смыслообразования с позиций его психологических и психофизиологических особенностей. Исследование психофизиологических коррелят процесса решения вербальных задач в ситуации смысловой инициации может в значительной мере расширить современные представления о психологической и психофизиологической природе смыслообразования. Такой подход позволит расширить и уточнить представления о психологических и психофизиологических механизмах, лежащих в основе мышления человека.

Целью проведенного исследования выступило изучение психологических и психофизиологических особенностей решения вербальных задач в ситуации смысловой инициации. Предметом - психологические особенности и биоэлектрическая активность коры головного мозга юношей и девушек в процессе решения вербальных задач в ситуации смысловой инициации. 
Гипотезы исследования были сформулированы следующим образом:

1. Характеристики биоэлектрической активности коры головного мозга могут отражать особенности процесса решения вербальных задач в ситуации смысловой инициации.

2. Характеристики биоэлектрической активности коры головного мозга в процессе решения вербальных задач в ситуации смысловой инициации у юношей и девушек могут различаться.

\section{Методы}

В исследовании приняли участие юноши и девушки в возрасте от 18 до 25 лет, средний возраст 19,8 \pm 1,6. Объем выборки - 52 человека (27 девушек, 25 юношей) с нормальным или скорректированным зрением.

Репрезентативность выборки определяется количеством участников эксперимента (необходимой и достаточной выборкой) и их искренним желанием принять участие в исследовании.

В ходе психофизиологического эксперимента использовался метод электроэнцефалографии (ЭЭГ). В начале эксперимента предъявлялись тренировочные задачи для обучения испытуемого правилам поведения в ходе записи ЭЭГ и адаптации к ситуации эксперимента. Далее участникам эксперимента в интерактивном режиме были визуально предъявлены вербальные задачи открытого типа (без заданного ответа или способа решения), моделирующие ситуацию смысловой инициации, а также контрольные задания - простые, эмоционально нейтральные логические задания.

Регистрация электроэнцефалограммы проводилась с использованием многоканального электроэнцефалографа «Нейровизор-136» (производства компании «МКС», Россия). Запись велась монополярно, стандартно, в 128 отведениях с двумя референтными ипсилатеральными ушными электродами (A1, А2). Для обработки записей ЭЭГ использовалось следующее программное обеспечение: Microsoft Excel, HBI database, WinEEG. Коррекция артефактов проводилась по независимым компонентам. Анализировались отрезки от начала до завершения выполнения тестовых (экспериментальных) и контрольных задач. Эпоха анализа составляла 1 секунду, полуперекрывание эпох - 50 \%, временное окно Ханна. Для дальнейшего анализа для каждого испытуемого и каждого задания были рассчитаны абсолютные значения мощностей в дельта $(\delta)$ - 1.5-4 Гц, тета $(\theta)$ - 4-8 Гц, альфа (a) - 8-13 Гц, бета1 ( $\beta 1)$ - 13-20 Гц, бета2 $(\beta 2)$ - 20-30 Гц и гамма $(\gamma 1)-30-50$ Гц диапазонах.

Сравнительный анализ достоверности различий показателей спектральной мощности и индекса по частотным диапазонам проводился с помощью многофакторного дисперсионного анализа ANOVA/MANOVA (repeated measures ANOVA) и сравнительного post-hoc анализа по критерию LSD Фишера. 


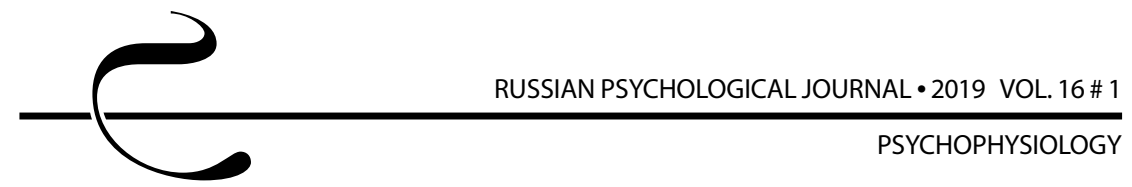

Обработка осуществлялась при помощи пакета компьютерных программ «STATISTICA 10.0».

\section{Результаты}

При анализе спектральной мощности основных ритмов ЭЭГ во время выполнения функциональных проб (при решении вербальных задач в ситуации смысловой инициации) в сравнении контрольными заданиями (при решении нейтральных простых вербальных мыслительных задач) удалось выявить статистически значимые различия в дельта- и альфа-диапазонах (рисунок 1).

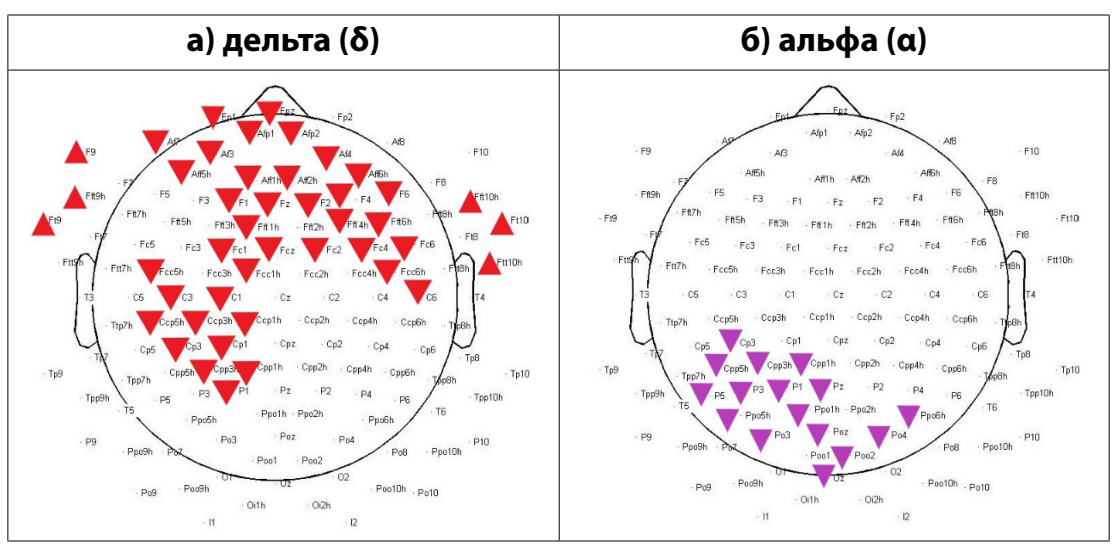

Рисунок 1. Различия по усредненным показателям мощности основных ритмов ЭЭГ во время выполнения тестовых и контрольных заланий $(p<0,05)$

Условные обозначения: а) различия дельта-диапазона; б) различия альфа-диапазона.

Figure 1. Differences in the average power values of EEG rhythms during the performance of test and control tasks $(p<0.05)$

Legend: a) differences in the delta frequency range; b) differences in the alpha frequency range.

При решении вербальных задач на смысл во фронтальных отведениях со сдвигом вправо (Fp1, FPz, Afp1, Afp2, Af7, Af3, Af4, Aff5, Aff1, Aff2, Aff6, F1, Fz, F2, F4, F6, Fft5, Fft1, Fft2, Fft4, Fft6, Fc1, Fcz, Fc2, Fc4, Fc6, Fcc5, Fcc1, Fcc6) и центральных отведениях со сдвигом влево (Сср3, Сср1, Ср3, Сp1, Сpp3, Сpp1) значимо снижается мощность дельта-ритма. При этом во фронтально-темпоральных (F9, FfT9, FfT10, Ft9, Ft10, Ftt10) отведениях билатерально мощность дельта-ритма возрастает. Отмечено снижение мощности альфа-диапазона в париетальной (Сp3, Сcp5, Сcp3, P5, P3, P1, Pz, Ppo5 Ppo1, Рpo6) и окципитальной (Po3, Poz, Po4, Poo2, Oz) областях. 
По относительному показателю мощности (индекс,\%) удалось выявить достоверные изменения во всех ритмических диапазонах электрической активности головного мозга при решении задач на смысл в сравнении с активностью во время решения контрольных заданий (рисунок 2).

При решении вербальных задач на смысл во фронтальных (Af3, Aff5, Aff6, F3, F1, Fz, F2, F4, Fft1, Fft2, Fft4, Fft6, Fc1, Fcz, Fc6, Fcc5, Fcc3) и центральных отведениях со сдвигом влево (C5, Tp7, Cp5, Cz) дельта-индекс значимо снижается, а во фронтально-темпоральных (F9, F10, FfT9, FfT10, Ft9, Ft10) отведениях билатерально - возрастает. Генерализованное снижение тета-индекса отмечается во фронтальных, фронтально-темпоральных и центральных отведениях. Индекс альфа-диапазона снижается в париетальной области слева (Р9, Р3, Рpo5) и отведениях Oz и P4, возрастает в центральных (Fcc3, Ccp4) и темпоральных отведениях справа (Ftt8, Ttp8). Индекс бета1 снижается в париетальных отведениях по центру со сдвигом к затылку (Cp1, Cpz, Cp2, Cpp3, Cpp1, Cpp2, Cpp4, P3, P1, Pz, P4, Рpo1, Рpo2, Рpo6, Рo3, Poz, Рo4). При этом в темпоральных отведениях справа отмечается возрастание индекса бета2 (Ttp7, Tp7, Cp5, Трp7) и гамма-индекса (T3, C5, Tp7, Cp5, Cp3).

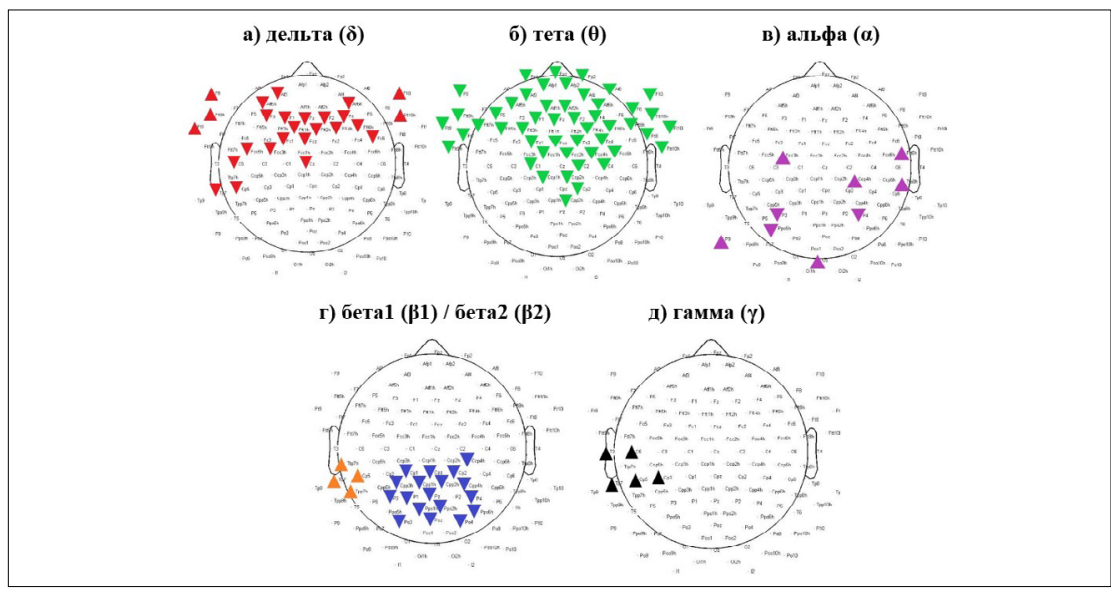

Рисунок 2. Различия по относительному показателю мощности (\%) основных ритмов ЭЭГ во время выполнения тестовых и контрольных заАаний $(p<0,05)$

Условные обозначения: а) различия дельта-индекса; б) различия тета-индекса; в) различия альфа-индекса; г) различия бета-индекса; д) различия гамма-индекса.

Figure 2. Differences in the relative power values (\%) of EEG rhythms during the performance of test and control tasks $(p<0.05)$

Legend: a) differences in the delta index; b) differences in the theta index; c) differences in the alpha index; d) differences in the beta index; e) differences in the gamma index. 
Таким образом, полученные результаты позволяют заключить, что биоэлектрическая активность коры головного мозга во время решения вербальных задач на смысл, в сравнении с контрольными пробами, характеризуется снижением мощности и индекса медленноволновой активности дельта-диапазона и индекса тета-диапазона во фронтальной и центральной областях, десинхронизацией альфа-ритма в затылочных областях со сдвигом влево и возрастанием альфа-индекса в темпоральной области справа. Кроме того, удалось установить, что при решении задач, инициирующих процесс смыслообразования, индекс бета1 снижается в париетальных отведениях по центру со сдвигом к затылку, а в темпоральных отведениях слева отмечается возрастание индекса бета2 и гамма-индекса.

Для того чтобы охарактеризовать частотно-пространственной функциональной организацией коры головного мозга в процессе решения вербальных задач в подгруппах юношей и девушек, сравнительный анализ мощностей и индексов основных ритмов ЭЭГ при помощи многофакторного дисперсионного анализа (Repeated measures ANOVA) был проведен по усредненным показателям спектральной мощности и индекса в очищенных от артефактов отрезках, соответствующих периодам решения тестовых (смысловых) и контрольных заданий в подгруппах юношей и девушек по-отдельности (девушки ТЕСТхКОНТРОЛЬ; юноши ТЕСТхКОНТРОЛЬ).

При анализе спектральной мощности основных ритмов ЭЭГ во время выполнения функциональных проб (тест) в сравнении с активностью при решении контрольных заданий в подгруппе девушек были выявлены статистически значимые различия в дельта- и альфа-диапазонах (рисунок 3).

Полученные в подгруппе девушек результаты в целом повторяют картину, полученную при сравнении мозговых коррелят решения вербальных задач на смысл и выполнения контрольных заданий во всей выборке. Во фронтальных отведениях со сдвигом вправо (Fp1, FPz, Afp1, Afp2, Af7, Af3, Af4, Af8, Aff5, Aff1, Aff2, Aff6, F1, Fz, F2, F4, Fft5, Fft1, Fft2, Fft4, Fft6, Fc1, Fcz, Fc2, Fc4, Fc6, Fcc1, Fcc2, Fcc4, Fcc6) и центральных отведениях со сдвигом влево (C3, C1, Cz, Ccp3, Ccp1, Cp3, Cp1, Cpz, Cpp1), а также в отведениях P1 и P6, мощность дельта-ритма значимо снижается. Во фронтально-темпоральных (F9, F10, F7, FfT9, FfT10, Ft9, Ft10, Ftt10) отведениях билатерально мощность дельта-ритма возрастает. Отмечено снижение мощности альфа-ритма в париетальной (Ср3, Cpz, Cpp5, Сpp3, Cpp1, P5, P3, P1, Pz, P2, Рpo5 Ppo1, Рpo2) и окципитальной (Ро3, Poz, Рo4, Oz) областях.

В результате сравнительного анализа индексов основных ритмов во время выполнения тестовых и контрольных заданий в подгруппе девушек выявлены достоверные изменения в дельта-, тета-, альфа- и бета-диапазонах (рисунок 4). 


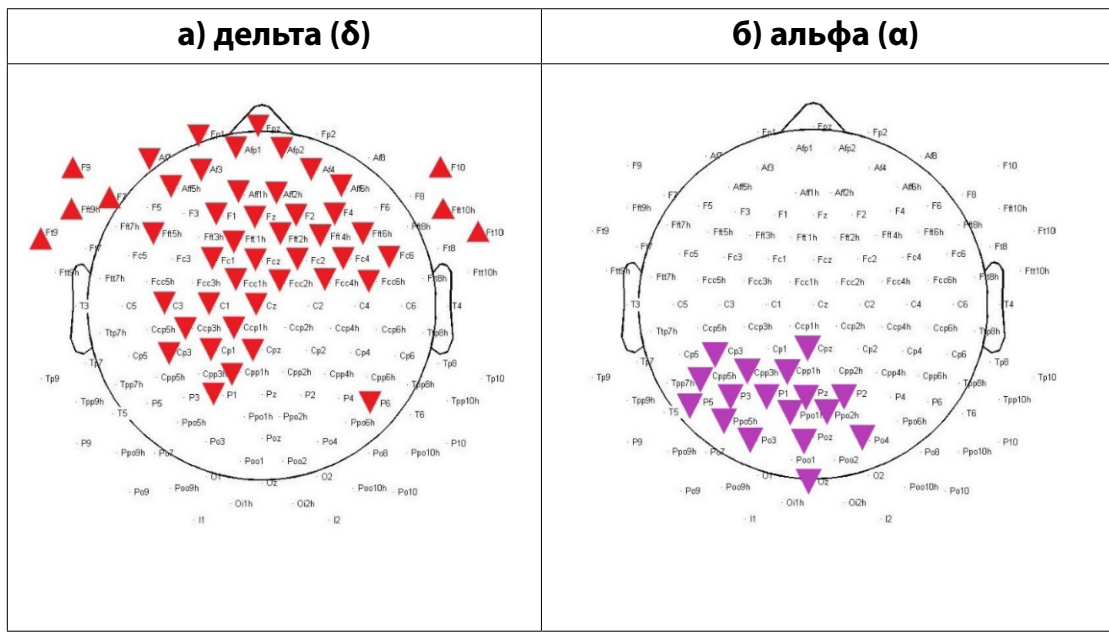

Рисунок 3. Различия по усреАненным показателям мощности основных ритмов ЭЭГ во время выполнения тестовых и контрольных заданий в полгруппе Аевушек $(\mathrm{p}<0,05)$

Условные обозначения: а) различия дельта-диапазона; б) различия альфа-диапазона.

Figure 3. Differences in the average power values of EEG rhythms during the performance of test and control tasks in the subgroup of girls $(p<0.05)$

Legend: a) differences in the delta frequency range; $b$ ) differences in the alpha frequency range.

При решении вербальных задач на смысл во фронтальных со сдвигом вправо (Fpz, Afp1, Afp2, Af3, Af4, Aff5, Aff1, Aff2, Aff6, F1, Fz, F2, F4, F6, F10, Fft1, Fft2, Fft4, Fft6, Fft10, Fcz, Fc2, Fc4, Fc6) и центральных отведениях со сдвигом влево (Fcc5, Fcc3, Fcc1, C3, C1, Cz, Ccp5, Ccp3, Ccp1, Cp5) дельта-индекс значимо снижается. Снижение тета-индекса отмечается во фронтальных отведениях со сдвигом вправо (Fpz, Afp1, Afp2, Af7, Af3, Af4, Af8, Aff5, Aff1, Aff2, Aff6, F9, F1, Fz, F2, F4, F6, F10, Fft1, Fft2, Fft4, Fft6, Fcz, Fc2, Fc4, Fc6), билатерально во фронтально-темпоральной области (Fft9, Fft8, Fft10, Ft9, Ft7, Ft10, Ftt10) и центральных отведениях (Fcc2, Fcc4, C2, Ccp2, Cp2). Индекс альфа-диапазона возрастает в темпоральных (Ftt8, Ft8, Ttp8), центральных (C6, Ccp4) и затылочных (P4, Oz, Oi2, Роо10, Ро10) отведениях правого полушария и отведении Р9. Индекс бета1 снижается в париетальных отведениях по центру со сдвигом к затылку (Ср1, Cpz, Cp2, Cpp3, Cpp1, Cpp2, Cpp4, P5, P3, P1, Pz, P2, P4, Ppo1, Ppo2, Ppo6, Рo3, Poz, Po4, Poo1). Индекс бета2 снижается в отведении Р9 и возрастает в темпоральных отведениях справа (Ftt7, Ttp7, Tp7). 

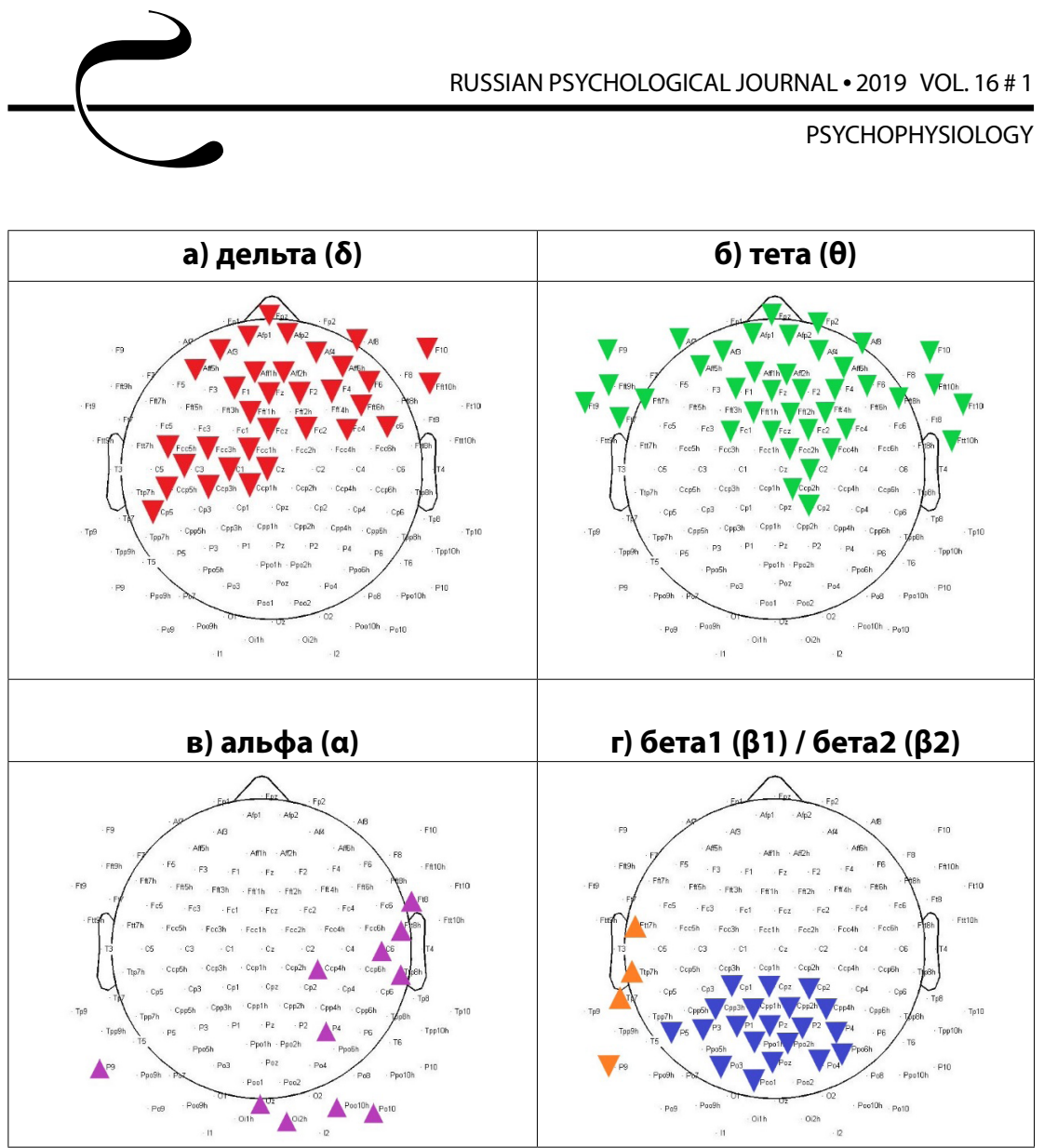

Рисунок 4. Различия индексов основных ритмов ЭЭГ во время выполнения

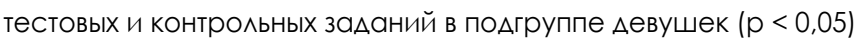

Условные обозначения: а) различия дельта-индекса; б) различия тета-индекса; в) различия альфа-индекса; г) различия бета-индекса.

Figure 4. Differences in the indices of EEG rhythms during the performance of test and control tasks in the subgroup of girls $(p<0.05)$

Legend: a) differences in the delta index; $b$ ) differences in the theta index; $c$ ) differences in the alpha index; d) differences in the beta index.

В подгруппе юношей анализ спектральной мощности основных ритмов ЭЭГ во время выполнения функциональных проб (тест), в сравнении с активностью при решении контрольных заданий, показал значительно меньше значимых различий (рисунок 5).

В затылочной области со сдвигом вправо удалось установить значимое снижение мощности дельта- (Рo3, Poo1, 01) и альфа- (Po3, Poz) диапазонов. Кроме того, у юношей мощность дельта-ритма возрастает во фронтально-темпоральных отведениях левого полушария (F9, Fft9, Ft9). 


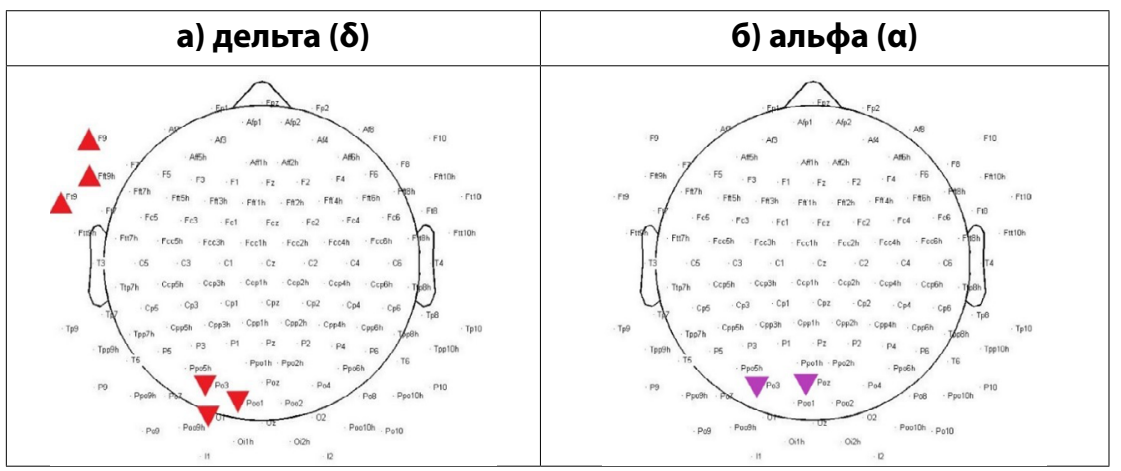

Рисунок 5. Различия по усреАненным показателям мощности основных ритмов ЭЭГ во время выполнения тестовых и контрольных заданий в полгруппе юношей $(p<0,05)$

Условные обозначения: а) различия дельта-диапазона; б) различия альфа-диапазона.

Figure 5. Differences in the average power values of EEG rhythms during the performance of test and control tasks in the subgroup of boys $(p<0.05)$

Legend: a) differences in the delta frequency range; $b$ ) differences in the alpha frequency range.

В результате сравнительного анализа индексов основных ритмов во время выполнения тестовых и контрольных заданий в подгруппе девушек выявлены достоверные изменения в тета-, альфа- и бета2-диапазонах (рисунок 6).

Снижение тета-индекса отмечается во фронтальных отведениях билатерально (Fp1, Fpz, Afp1, Afp2, Af3, Af4, Aff1, Aff2, F9, F5, F1, F6, F10, Fft9, Fft5, Fft1, Fft10, Ft9, Ft10, Fc1, Fcc1). В левом полушарии в париетальных отведениях P3 и Рро5 снижается индекс альфа-ритма и возрастает индекс бета2 в затылочной области (Ро7, 01, Oi1, I1).

Таким образом, полученные результаты позволяют заключить, что характеристики биоэлектрической активности коры головного мозга в процессе решения вербальных задач в ситуации смысловой инициации у юношей и девушек различаются.

В подгруппе девушек биоэлектрическая активность коры головного мозга во время решения вербальных задач на смысл, в сравнении с контрольными пробами, характеризуется снижением мощности и индекса медленноволновой активности дельта-диапазона и индекса тета-ритма во фронтальной и центральной областях, десинхронизацией альфа-ритма в затылочных областях со сдвигом влево и возрастанием альфа-индекса в темпоральной и париетальной областях правого полушария. Также удалось установить, 


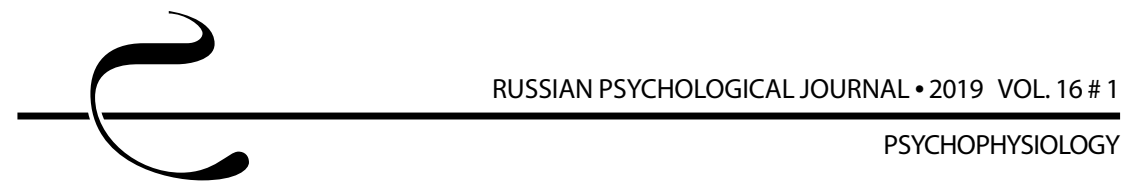

что при решении задач на смысл индекс бета1 снижается в париетальных отведениях по центру со сдвигом к затылку, а в темпоральных отведениях справа отмечается возрастание индекса бета2.

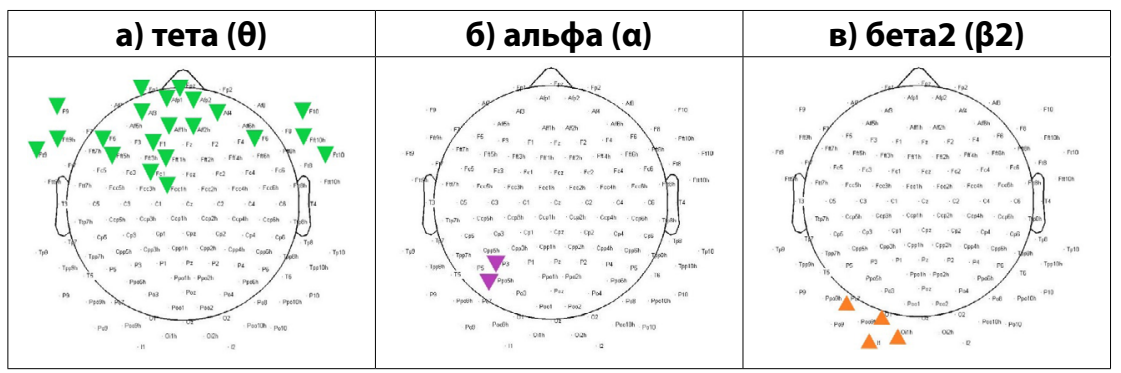

Рисунок 6. Различия инАексов основных ритмов ЭЭГ во время выполнения тестовых и контрольных заланий в полгруппе юношей $(p<0,05)$

Условные обозначения: а) различия тета-индекса; б) различия альфа-индекса; в) различия бета-индекса.

Figure 6. Differences in the indices of EEG rhythms during the performance of test and control tasks in the subgroup of boys $(p<0.05)$

Legend: a) differences in the theta index; $b$ ) differences in the alpha index; c) differences in the beta index.

В подгруппе юношей частотно-пространственная функциональная организация коры головного мозга в процессе решения вербальных задач, в сравнении с контрольными заданиями, отличается генерализованным фронтальным снижением тета-индекса, повышением мощности дельта-ритма во фронтальной области левого полушария, а также десинхронизацией альфа-ритма и возрастанием высокочастотного бета2-ритма париетально-окципитальной области слева.

\section{Обсуждение результатов}

Полученные результаты свидетельствуют о том, что на фоне общего повышения медленноволновой активности во фронтальных и центральных областях, при сравнении с контрольными пробами, эта активность значимо снижается. В научной литературе снижение медленноволновой принято считать показателем более высокого уровня активации [35, 36]. Однако снижение мощности и длительности синхронизации в дельта-диапазоне при решении тестовых задач может также свидетельствовать о меньшей концентрации внимания респондентов на условии задачи [37, 38]. Центрально-лобный тета-ритм традиционно связывают с общим повышением активации, концентрации внимания, усилением ориентировочной реакции и эффективности переработки информации [39, 
$40,41,42]$. Соответственно, снижение индекса тета-ритма при решении задач на смысл во фронтальных и центральных областях может свидетельствовать о снижении когнитивного напряжения и уровня активации передних отделов коры. При этом, одновременное снижение дельта- и тета-индекса может быть рассмотрено неоднозначно. С одной стороны, большая активация может отражать усиление сознательного контроля, с другой - снижение концентрации внимания респондентов на условии задачи $[36,38,43,44]$.

Выраженная десинхронизация альфа-ритма, вероятно, может быть рассмотрена как повышение активности зоны ТПО левого полушария, при этом снижение индекса бета-1 окципитальной области будет отражать переход от образного мышления к логико-семантическому мультимодальному синтезу во время решения задач, направленных на инициацию личностного смысла. Данное предположение может быть подкреплено как данными психофизиологических исследований, указывающих на роль зоны ТПО в обеспечении мыслительной деятельности [31, 45], так и нейропсихологическими данными о развитии «семантической афазии» при поражении зоны ТПО левого (у правшей) полушария [46]. Что касается длительности синхронизации бета-ритма, можно предположить, что увеличение индекса бета-2 в темпоральной области слева отражает увеличение активности центров переработки вербальной информации. Однако необходимо отметить, что пространственная локализация этих различий соответствует центральной части поясной извилины и сопровождается увеличением индекса гамма-ритма. Преобладание высокочастотной ритмики связывают с увеличением нагрузки на рабочую память [47], а также с повышением субъективной значимости стимула [26]. Соответственно, можно предположить, что повышение индекса бета2- и гамма-ритмов в левой темпоральной области при решении задач на смысл отражает не только характер, но и эмоциогенность стимула, а также сопровождается большей нагрузкой на рабочую память.

В современной литературе все больше исследователей приходит к выводу о том, что при внешнем сходстве поведенческих проявлений и равной эффективности когнитивной деятельности мозговая активность мужчин и женщин может принципиально отличаться [28, 31, 48, 49, 50]. Данные, полученные в ходе сравнения усредненных показателей мощности и относительного показателя представленности ритмических компонентов в ЭЭГ при решении тестовых и контрольных задач в подгруппах юношей и девушек, позволяют заключить, что характеристики биоэлектрической активности коры головного мозга в процессе решения вербальных задач в ситуации смысловой инициации у юношей и девушек различаются.

В подгруппе девушек биоэлектрическая активность коры головного мозга во время решения вербальных задач на смысл, в сравнении с контрольными пробами, характеризуется снижением мощности и индекса медленноволновой 
активности дельта-диапазона и индекса тета-ритма во фронтальной и центральной областях, десинхронизацией альфа-ритма в затылочных областях со сдвигом влево, что сходно с картиной различий во всей выборке. В подгруппе девушек также удалось установить повышение альфа-индекса в темпоральной и париетальной областях правого полушария, снижение индекса бета1 в париетальных отведениях со сдвигом к затылку и возрастание индекса бета2 в правой темпоральной области. Полученные результаты согласуются с данными исследований вербальной творческой деятельности О. М. Разумниковой и И. В. Тарасовой $[22,31]$ и данными о преобладании левополушарной активности у женщин [51]. Кроме того, показано, что с активностью преимущественно левого полушария связана работа семантической памяти $[21,52]$. Другими словами, наши результаты свидетельствуют о том, что процесс продумывания ответа на задачи, инициирующие личностный смысл, характеризуется выраженным смещением фокуса активности в левое полушарие и требует большего вовлечения семантической памяти.

В подгруппе юношей частотно-пространственная функциональная организация коры головного мозга в процессе решения смысловых задач, в сравнении с контрольными заданиями, отличается генерализованным фронтальным снижением тета-индекса, повышением мощности дельта-диапазона во фронтальной области левого полушария, а также десинхронизацией альфа-ритма и возрастанием высокочастотного бета2-ритма париетально-окципитальной области слева. Необходимо отметить, что сходная картина в подгруппах наблюдается только по индексу тета-ритма. Во всех других ритмических диапазонах различий, во-первых, значительно меньше, во-вторых, они имеют другую пространственную локализацию. Согласно описанным результатам, в группе юношей решение вербальных задач на смысл также характеризуется большей активацией задних отделов коры левого полушария. Схожие данные были получены при исследовании работы мозга во время решения вербальных задач и исследовании мозговых механизмов понятийного мышления $[17,29]$. Вероятно, увеличение длительности синхронизации бета-2 может объясняться привлечением образов долговременной памяти.

\section{Заключение}

Таким образом, анализ полученных результатов позволяет говорить о том, что особенности процесса смыслообразования во время решения вербальных задач в ситуации смысловой инициации характеризуются особой частотно-пространственной организацией биоэлектрической активности коры головного мозга.

Биоэлектрическая активность коры головного мозга во время решения вербальных задач в ситуации смысловой инициации, в сравнении с контрольными 
пробами, характеризуется снижением мощности и индекса медленноволновой активности дельта-диапазона и индекса тета-диапазона во фронтальной и центральной областях, десинхронизацией альфа-ритма в затылочных областях со сдвигом влево и возрастанием альфа-индекса в темпоральной области справа ( $\mathrm{p}<0,05)$. Кроме того, удалось установить, что при решении задач, инициирующих процесс смыслообразования, индекс бета1 снижается в париетальных отведениях по центру со сдвигом к затылку, а в темпоральных отведениях слева отмечается возрастание индекса бета2 и гамма-индекса $(p<0,05)$. Полученные результаты, по-видимому, свидетельствуют о повышении функциональной активности фронтальных и центральных областей мозга, а также зоны ТПО левого полушария, и могут отражать эмоциогенность стимула и отказ от стереотипного способа решения задач на смысл.

Специфика перехода от простой мыслительной деятельности к решению вербальных задач в ситуации смысловой инициации различается у юношей и девушек. В подгруппе девушек биоэлектрическая активность коры головного мозга во время решения вербальных задач в ситуации смысловой инициации, в сравнении с контрольными пробами, характеризуется снижением мощности и индекса медленноволновой активности дельта-диапазона и индекса тета-ритма во фронтальной и центральной областях, десинхронизацией альфа-ритма в затылочных областях со сдвигом влево и возрастанием альфа-индекса в темпоральной и париетальной областях правого полушария $(p<0,05)$. Также удалось установить, что при решении задач в ситуации смысловой инициации индекс бета1 снижается в париетальных отведениях по центру со сдвигом к затылку, а в темпоральных отведениях справа отмечается возрастание индекса бета2 ( $<<0,05)$. В подгруппе юношей суммарная электрическая активность коры головного мозга в процессе решения смысловых задач, в сравнении с контрольными заданиями, характеризуется генерализованным снижением тета-индекса и повышением мощности дельта-ритма во фронтальной области левого полушария, а также десинхронизацией альфа-ритма и возрастанием бета2-ритма париетально-окципитальной области слева $(p<0,05)$. Описанные различия, по-видимому, свидетельствуют о том, что процесс смыслообразования у девушек в большей степени опирается на мультимодальную информацию и выстраивание семантических отношений. В то время как юноши в большей степени опираются на наглядные образы долговременной памяти.

Результаты исследования могут найти практическое применение в сфере образования при разработке программ, направленных на развитие личностного потенциала, стимуляцию познавательной и творческой активности учащихся, а также в практической работе психологов при разработке методик нейропсихологической коррекции и реабилитации обучающихся с особыми образовательными потребностями и трудностями в обучении. 


\section{Литература}

1. Асмолов А. Г. По ту сторону сознания: методологические проблемы неклассической психологии. М.: Смысл, 2002. 480 с.

2. Леонтьев Д. А. Психология смысла: природа строение и динамика смысловой реальности. 3-е изд., доп. М.: Смысл, 2007. 511 с.

3. Строй Г.В. Генезис понятия «личностный смысл» в психологии // Образование. Наука. Научные Кадры. 2011. № 3. С. 212-214.

4. Абакумова И. В., Ермаков П. Н., Фоменко В. Т. Новодидактика. Кн. 2. Образовательные технологии: новые ракурсы. М.: Кредо, 2013. 122 с.

5. Братусь Б. С. Проблема возвращения категории «души» в научную психологию // Национальный психологический журнал. 2014. № 3 (15). С. 3-12.

6. Горская Н. Е. Современные подходы к проблеме смысла и смыслообразования в учебном процессе // Социальная компетентность. 2017. T. 2, № 4. С. 46-51.

7. Абакумова И. В., Годунов М. В., Енин А. Л., Генердукаева 3. Ш. Стратегии смыслообразования: современные представления в работах отечественных исследователей: учебное пособие. М.: КРЕДО, 2016. 36 с.

8. Годунов М. В., Елагина М. Ю., Белова Е. В. Исследование личностных профилей полярных стратегий смыслообразования // Российский психологический журнал. 2017. T. 14, № 3. C. 30-47. DOI: 10.21702/rpj.2017.3.2

9. Почтарева Е. Ю. Ценностно-смысловая сфера личности: сущность, детерминанты, механизмы развития // Вестник Пермского университета. Философия. Психология. Социология. 2017. № 4. URL: https://cyberleninka. ru/article/n/tsennostno-smyslovaya-sfera-lichnosti-suschnost-determinantymehanizmy-razvitiya (дата обращения: 17.02.2018).

10. Ермаков П. Н., Абакумова И. В. Формирование идеологических установок и мировоззренческих ценностей личности в контексте теории смысла и смыслообразования // От истоков к современности. 130 лет организации психологического общества при Московском университете: сборник материалов юбилейной конференции в 5 томах. М.: Когито-Центр, 2015. С. 274-277.

11. Зорина Е. С., Зеленов А. А. Психологические основы смыслотехник как современных образовательных методов // Российский психологический журнал. 2016. T. 13, № 1. C. 76-84. DOI: 10.21702/rpj.2016.1.6

12. Выготский Л. С. Мышление и речь. 5-е изд., испр. М.: Лабиринт, 1999. 352 с.

13. Бабаева Ю. Д., Березанская Н. Б., Васильев И. А., Войскунский А. Е., Корнилова Т. В. Смысловая теория мышления // Вестник Московского университета. Серия 14: Психология. 2008. № 2. URL: https://cyberleninka. ru/article/n/smyslovaya-teoriya-myshleniya (дата обращения: 13.02.2018). 
14. Иваницкий А. М., Ильюченок И. Р. Картирование биопотенциалов при решении вербальной задачи // Журнал Высшей Нервной Деятельности. 1992. T. 42, № 4. С. 627-635.

15. Pulvermūller F., Keil A., Elbert T. Hight-frequency brain activity: perception or active memory? // Trends in Cognitive Sciences. 1999. Vol. 3, Issue 7. P. 250-252. DOI: 10.1016/S1364-6613(99)01344-3

16. von Stein A., Rappelsberger P., Sarnthein J., Petsche H. Synchronization Between Temporal and Parietal Cortex During Multimodal Object Processing in Man // Cerebral Cortex. 1999. Vol. 9, № 2. P. 137-150.

17. Воробьева Е. В., Харитонова И. Ю. Генотип-средовые детерминанты мощности ритмических составляющих ЭЭГ при вербально-ассоциативной деятельности // Новые исследования. 2010. № 2 (23). С. 5-16.

18. Popova T. V., Koryukalov Y. I., Kourova O. G. Some of the Brain Mechanisms of the State of Induced Relaxation // Advances in Bioscience and Bioengineering. 2014. Vol. 2, Issue 2. P. 8-13. DOI: $10.11648 /$ j.abb.20140202.11

19. Milz P., Faber P. L., Lehmann D. et al. The functional significance of EEG microstates. Associations with modalities of thinking // Neurolmage. 2016. Vol. 125. P. 643-656. DOI: 10.1016/j.neuroimage.2015.08.023

20. Wang M., Hao N., Ku Y., Grabner R. H., Fink A. Neural correlates of serial order effect in verbal divergent thinking // Neuropsychologia. 2017. Vol. 99. P. 92-100. DOI: 10.1016/j.neuropsychologia.2017.03.001

21. Jung-Beeman M., Bowden E. M., Haberman J., Frymiare J. L., Arambel-Liu S., Greenblatt R., Reber P. J., Kounios J. Neural Activity When People Solve Verbal Problems with Insight // PLOS Biol. 2004. 2 (4). e97. DOI: 10.1371/journal. pbio.0020097

22. Тарасова И. В., Разумникова О. М., Вольф Н. В. Связь изменений мощности ЭЭГ с инструкцией, стимулирующей творческое мышление у мужчин и женщин // Журнал высшей нервной деятельности. 2006. Т. 56, № 2. С. 611-617.

23. Дикая Л. А., Шиварева С. Ю. Мозговая организация функциональных связей у лиц, занимающихся научной и литературной профессиональной деятельностью, при решении вербальных творческих задач // Северо-Кавказский психологический вестник. 2011. № 9/1. С. 18-21.

24. Ермаков П. Н., Скиртач И. А., Ковш Е. М. Особенности частотно-пространственной организации коры мозга у музыкантов во время создания импровизации на основе стимульного материала с агрессивным и миролюбивым «характерами» // Российский психологический журнал. 2015. T. 12, № 4. C. 127-136. DOI: 10.21702/rpj.2015.4.10

25. Grossman M., Smith E. E., Koening P. et al. The neural basis for categorization in semantic memory // Neurolmage. 2002. Vol. 17. P. 1549-1561. 
26. Jensen O., Kaiser J., Lachaux J.-P. Human gamma frequency oscillations associated with attention and memory // Trends in Neurosciences. 2007. Vol. 30, Issue 7. P. 317-324. DOI: 10.1016/j.tins.2007.05.001

27. Зачченко А. А., Картавенко М. В. Особенности ритмической активности головного мозга при актуализации модально-специфических видов памяти // Известия ЮФУ. Технические науки. 2010. № 10 (111). С. 167-174.

28. Reed J. L., Gallagher N. M., Sullivan M., Callicott J. H., Green A. E. Sex differences in verbal working memory performance emerge at very high loads of common neuroimaging tasks // Brain and Cognition. 2017. Vol. 113. P. 56-64. DOI: $10.1016 /$ j.bandc.2017.01.001

29. Голованова И. В., Балин В.Д. Психофизиологические корреляты успешного и неуспешного решения мыслительных задач и их связь с пониманием инструкций // Вестник ЮУрГУ. Серия: Психология. 2017. № 4. URL: https:// cyberleninka.ru/article/n/psihofiziologicheskie-korrelyaty-uspeshnogo-ineuspeshnogo-resheniya-myslitelnyh-zadach-i-ih-svyaz-s-ponimanieminstruktsiy (дата обращения: 17.07.2018).

30. Старченко М. Г.Исследование нейрофизиологического обеспечения параметров вербальной креативности-беглости, гибкости, оригинальности. Сообщение 2. - Данные позитронно-эмиссионной томографии // Международный научно-исследовательский журнал. 2018. № 2 (68). С. 13-15.

31. Разумникова О. М. Частотно-пространственная организация активности коры мозга человека при конвергентном и дивергентном мышлении в зависимости от фактора пола: Сообщение І. Анализ мощности ЭЭГ // Физиология человека. 2004. Т. 30, № 6. С. 17-27.

32. Бехтерева Н. П., Данько С. Г., Старченко М. Г., Шемякина Н. В. Электроэнцефалографические исследования креативного мышления как когнитивно-специфического функционального состояния: Материалы Международной конференции «Творчество: взгляд с разных сторон». Москва-Звенигород, 2005. С. 48-54.

33. Дикая Л. А., Дикий И. С. Творческий мозг: монография. Ростов-на-Дону: Изд-во ЮФУ, 2015. 218 с.

34. Fink A., Benedek M., Koschutnig K., Papousek l., Weiss E. M., Bagga D., SchöpfV. Modulation of resting-state network connectivity by verbal divergent thinking training // Brain and Cognition. 2018. Vol. 128. P. 1-6. DOI: 10.1016/j. bandc.2018.10.008

35. Кирой В. Н., Ермаков П. Н. Электроэнцефалограмма и функциональные состояния человека. Ростов-на-Дону: Изд-во Рост. ун-та, 1998. 264 с.

36. Кропотов Ю. Д. Количественная ЭЭГ, когнитивные вызванные потенциалы мозга человека и нейротерапия: пер. с англ. / под ред. В. А. Пономарева. Донецк: Издатель Заславский А. Ю., 2010. 512 с. 
37. Knyazev G. G. EEG delta oscillations as a correlate of basic homeostatic and motivational processes // Neuroscience \& Biobehavioral Reviews. 2012. Vol. 36, Issue 1. P. 677-695. DOI: 10.1016/j.neubiorev.2011.10.002

38. Harmony $T$. The functional significance of delta oscillations in cognitive processing // Frontiers in Integrative Neuroscience. 2013. Vol. 7. DOI: 10.3389/ fnint.2013.00083

39. Афтанас Л. И., Рева Н. В., Варламов А. А., Павлов С. В., Махиев В. П. Анализ вызванной синхронизации и десинхронизации ЭЭГ при эмоциональной активации у человека: временные и топографические характеристики // Журнал высшей нервной деятельности. 2003. Т. 53, № 4. С. 485-494.

40. Разумникова О. М., Вольф Н. В., Тарасова И. В. Влияние мотивации на частотно-пространственные характеристики активности коры головного мозга при выполнении образных и вербальных творческих заданий // Журнал высшей нервной деятельности. 2008. Т. 58, №5. С. 540-553.

41. Danilova N. N., Strabykina E. A. Frequency-selective generators of oscillatory brain activity allow identifying processes of a working memory // International Journal of Psychophysiology. 2010. Vol. 77, Issue 3. P. 208. DOI: 10.1016/j.ijpsycho.2010.06.011

42. Данилова Н. Н., Ушакова Т. Н., Волков Г. В., Плигина А. М., Страбыкина Е. А. Отображение семантических категорий в электрической активности мозга // Экспериментальная психология. 2013. Т. 6, № 4. С. 5-21.

43. Posner M. I., Petersen S. E. The Attention System of the Human Brain // Annual Review of Neuroscience. 1990. Vol. 13. P. 25-42.

44. Fan J., McCandliss B. D., Sommer T., Raz A., Posner M. I. Testing the Efficiency and Independence of Attentional Networks // Journal of Cognitive Neuroscience. 2002. Vol. 14, Issue 3. P. 340-347. DOI: 10.1162/089892902317361886

45. Mihov K. M., Denzler M., Förster J. Hemispheric specialization and creative thinking: a meta-analytic review of lateralization of creativity // Brain and Cognition. 2010. Vol. 72, № 3. P. 442-448.

46. Лурия А. Р. Основы нейропсихологии. М.: Академия, 2003. 384 с.

47. Думенко В. Н., Козлов М. К. Динамика мощности гамма-активности ЭЭГ вызванных ответов на лицевую экспрессию в условиях нагрузки на рабочую память // Журнал высшей нервной деятельности. 2012.62 (1). С. 20-32.

48. Halpern D. F. Sex differences in cognitive abilities. 3rd ed. Mahwah; N.Y.; London: Lawrence Erlbaum Ass. Publ., 2000. 420 p.

49. Thomsen T., Hugdahl K., Ersland L. et al. Functional magnetic resonance imaging (fMRI) study of sex differences in a mental rotation task // Medical Science Monitor. 2000.6 (6). P. 1186-1196.

50. Fink A., Neubauer A. C. EEG alpha oscillations during the performance of verbal creativity tasks: Differential effects of sex and verbal intelligence // 
International Journal of Psychophysiology. 2006. Vol. 62, Issue 1. P. 46-53. DOI: $10.1016 /$ j.ijpsycho.2006.01.001

51. Вольф Н. В., Разумникова О. М., Брызгалов А. О., Онищенко М. А., Лапина Е. Ю. Нейрофизиологический анализ механизмов полового диморфизма когнитивных стратегий: роль селективных процессов // Бюллетень CO PAMH. 2010. T. 30, № 4. C. 6-13.

52. Klimesch W. EEG alpha and theta oscillations reflect cognitive and memory performance: a review and analysis // Brain Research Reviews. 1999. Vol. 29, Issues 2-3. P. 169-195. DOI: 10.1016/S0165-0173(98)00056-3

\section{References}

1. Asmolov A. G. Po tu storonu soznaniya: metodologicheskie problemy neklassicheskoipsikhologii [On the other side of consciousness: Methodological problems of nonclassical psychology]. Moscow, Mysl' Publ., 2002. 480 p.

2. Leont'ev D. A. Psikhologiya smysla: priroda stroenie i dinamika smyslovoi real'nosti [Psychology of meaning: Nature, structure, and dynamics of semantic reality]. Moscow, Smysl Publ., 2007. 511 p.

3. Stroi G. V. Genesis of the concept of personal meaning in psychology. Obrazovanie. Nauka. Nauchnye Kadry-Education. Science. Scientific Personnel, 2011, no. 3, pp. 212-214 (in Russian).

4. Abakumova I. V., Ermakov P. N., Fomenko V. T. Novodidaktika. Kn. 2. Obrazovatel'nye tekhnologii: novye rakursy [New-didactics. Book 2. Educational technologies: new viewpoints]. Moscow, Kredo Publ., 2013. 122 p.

5. Bratus' B. S. The problem of restoring the category of soul in academic psychology. Natsional'nyi psikhologicheskii zhurnal - National Psychological Journal, 2014, no. 3 (15), pp. 3-12 (in Russian).

6. Gorskaya N. E. Modern approaches to meaning and meaning-building issues in the educational process. Sotsial'naya kompetentnost'-Social Competence, 2017, V. 2, no. 4, pp. 46-51 (in Russian).

7. Abakumova I. V., Godunov M. V., Enin A. L., Generdukaeva Z. Sh. Strategii smysloobrazovaniya: sovremennye predstavleniya v rabotakh otechestvennykh issledovatelei [Meaning-building strategies: Modern ideas in the works of domestic researchers]. Moscow, Kredo Publ., 2016. 36 p.

8. Godunov M. V., Elagina M. J., Belova E. V. Studying the personal profiles of the polar meaning-building strategies. Rossiiskii psikhologicheskii zhurnal Russian Psychological Journal, 2017, V. 14, no. 3, pp. 30-47 (in Russian). DOI: 10.21702/rpj.2017.3.2

9. Pochtareva E. Yu. Value-meaning sphere of personality: Essence, determinants, and mechanisms of development. Vestnik Permskogo universiteta. Filosofiya. Psikhologiya. Sotsiologiya - Perm University Herald. Series: 
Philosophy. Psychology. 2017. No. 4. Available at: https://cyberleninka.ru/ article/n/tsennostno-smyslovaya-sfera-lichnosti-suschnost-determinantymehanizmy-razvitiya (Accessed 17 February 2018).

10. Ermakov P. N., Abakumova I. V. Formirovanie ideologicheskikh ustanovok i mirovozzrencheskikh tsennostei lichnosti v kontekste teorii smysla i smysloobrazovaniya [Formation of ideological attitudes and worldview values of individuals in the context of the theory of meaning and meaningbuilding]. Ot istokov k sovremennosti. 130 let organizatsii psikhologicheskogo obshchestva priMoskovskom universitete:sbornikmaterialov yubileinoi konferentsii $v 5$ tomakh [Proc. the anniversary conference "From the beginning to the present: 130 years of the organization of the psychological society at Moscow University: in 5 volumes"]. Moscow, Kogito-Tsentr Publ., 2015, pp. 274-277.

11. Zorina E. S., Zelenov A. A. Psychological bases of meaning techniques as modern educational methods. Rossiiskii psikhologicheskiizhurnal-Russian Psychological Journal, 2016, V. 13, no. 1, pp. 76-84 (in Russian). DOI: 10.21702/rpj.2016.1.6

12. Vygotskii L. S. Myshlenie i rech' [Thinking and speech]. Moscow, Labirint Publ., 1999. 352 p.

13. Babaeva Yu. D., Berezanskaya N. B., Vasil'ev I. A., Voiskunskii A. E., Kornilova T.V. Meaning theory of thinking. Vestnik Moskovskogo universiteta. Seriya 14: Psikhologiya - The Moscow University Herald. Series 14: Psychology, 2008, no. 2. Available at: https://cyberleninka.ru/article/n/smyslovaya-teoriyamyshleniya (Accessed 13 February 2018).

14. Ivanitskii A. M., Il'yuchenok I. R. Mapping of brain biopotentials during solution of verbal tasks. Zhurnal Vysshei Nervnoi Deyatel'nosti - Journal of Higher Nervous Activity, 1992, V. 42, no. 4, pp. 627-635 (in Russian).

15. Pulvermüller F., Keil A., Elbert T. Hight-frequency brain activity: perception or active memory? Trends in Cognitive Sciences, 1999, V. 3, Issue 7, pp. 250-252. DOI: 10.1016/S1364-6613(99)01344-3

16. von Stein A., Rappelsberger P., Sarnthein J., Petsche H. Synchronization between temporal and parietal cortex during multimodal object processing in man. Cerebral Cortex, 1999, V. 9, no. 2, pp. 137-150.

17. Vorobyeva E. V., Kharitonova I. Yu. Genotype-environmental determinants of the power of EEG rhythmic components in verbal-associative activity. Novye issledovaniya - New Studies, 2010, no. 2 (23), pp. 5-16 (in Russian).

18. Popova T. V., Koryukalov Y. I., Kourova O. G. Some of the brain mechanisms of the state of induced relaxation. Advances in Bioscience and Bioengineering, 2014, V. 2, Issue 2, pp. 8-13. DOI: 10.11648/j.abb.20140202.11

19. Milz P., Faber P. L., Lehmann D. et al. The functional significance of EEG microstates. Associations with modalities of thinking. Neurolmage, 2016, V. 125, pp. 643-656. DOI: 10.1016/j.neuroimage.2015.08.023 
20. Wang M., Hao N., Ku Y., Grabner R. H., Fink A. Neural correlates of serial order effect in verbal divergent thinking. Neuropsychologia, 2017, V. 99, pp. 92-100. DOI: 10.1016/j.neuropsychologia.2017.03.001

21. Jung-Beeman M., Bowden E. M., Haberman J., Frymiare J. L., Arambel-Liu S., Greenblatt R., Reber P. J., Kounios J. Neural activity when people solve verbal problems with insight. PLOS Biol, 2004, 2 (4), e97. DOI: 10.1371/journal. pbio.0020097

22. Tarasova I. V., Razumnikova O. M., Vol'f N. V. Relationship between changes in the EEG power and instructions stimulating creative thinking in men and women. Zhurnal Vysshei Nervnoi Deyatel'nosti - Journal of Higher Nervous Activity, 2006, V. 56, no. 2, pp. 611-617 (in Russian).

23. Dikaya L. A., Shivareva S. Yu. Brain organization of functional associations in persons engaged in scientific and literary professional activity during the performance of verbal creative tasks. Severo-Kavkazskiipsikhologicheskii vestnikNorth-Caucasian Psychological Bulletin, 2011, no. 9/1, pp. 18-21 (in Russian).

24. Ermakov P. N., Skirtach I. A., Kovsh E. M. Features of frequency-spatial organization of the cerebral cortex among musicians during the creation of improvisation based on stimulus materials with aggressive and peaceful characters. Rossiiskii psikhologicheskii zhurnal - Russian Psychological Journal, 2015, V. 12, no. 4, pp. 127-136 (in Russian). DOI: 10.21702/rpj.2015.4.10

25. Grossman M., Smith E. E., Koening P. et al. The neural basis for categorization in semantic memory. Neurolmage, 2002, V. 17, pp. 1549-1561.

26. Jensen O., Kaiser J., Lachaux J.-P. Human gamma frequency oscillations associated with attention and memory. Trends in Neurosciences, 2007, V. 30, Issue 7, pp. 317-324. DOI: 10.1016/j.tins.2007.05.001

27. Zaichenko A. A., Kartavenko M. V. Features of rhythmic activity of the brain during the actualization of modal-specific types of memory. Izvestiya YuFU. Tekhnicheskie nauki - Izvestiya SFedU. Engineering Sciences, 2010, no. 10 (111), pp. 167-174 (in Russian).

28. Reed J. L., Gallagher N. M., Sullivan M., Callicott J. H., Green A. E. Sex differences in verbal working memory performance emerge at very high loads of common neuroimaging tasks. Brain and Cognition, 2017, V. 113, pp. 56-64. DOI: 10.1016/j.bandc.2017.01.001

29. Golovanova I. V., Balin V. D. Psychophysiological correlates of successful and unsuccessful problem solving and its connection with instructions understanding. Vestnik YuUrGU. Seriya: Psikhologiya - Bulletin of the South Ural State University. Series: Psychology, 2017, no. 4. Available at: https:// cyberleninka.ru/article/n/psihofiziologicheskie-korrelyaty-uspeshnogo-ineuspeshnogo-resheniya-myslitelnyh-zadach-i-ih-svyaz-s-ponimanieminstruktsiy (Accessed 17 July 2018). 
30. Starchenko M. G. Investigation of neurophysiological support of parameters of verbal creativity-fluency, flexibility, and originality. Message 2: Data of positron emission tomography. Dannye pozitronno-emissionnoi tomografii International Research Journal, 2018, no. 2 (68), pp. 13-15 (in Russian).

31. Razumnikova O. M. Frequency-spatial organization of human cerebral cortex activity in convergent and divergent thinking depending on sex: Message 1: EEG power analysis. Fiziologiya cheloveka - Human Physiology, 2004, V. 30, no. 6, pp. 17-27 (in Russian).

32. Bekhtereva N. P., Dan'ko S. G., Starchenko M. G., Shemyakina N. V. Elektroentsefalograficheskie issledovaniya kreativnogo myshleniya kak kognitivnospetsificheskogo funktsional'nogo sostoyaniya [Electroencephalographic studies of creative thinking as a cognitive-specific functional state]. Materialy Mezhdunarodnoi konferentsii "Tvorchestvo: vzglyad s raznykh storon" [Proc. the International Conference "Creativity: Views from different angles"]. Moscow-Zvenigorod, 2005, pp. 48-54.

33. Dikaya L. A., Dikii I. S. Tvorcheskii mozg [Creative brain]. Rostov-on-Don, SFU Publ., 2015. 218 p.

34. Fink A., Benedek M., Koschutnig K., Papousek I., Weiss E. M., Bagga D., Schöpf V. Modulation of resting-state network connectivity by verbal divergent thinking training. Brain and Cognition, 2018, V. 128, pp. 1-6. DOI: 10.1016/j.bandc.2018.10.008

35. Kiroi V. N., Ermakov P. N. Elektroentsefalogramma i funktsional'nye sostoyaniya cheloveka [Electroencephalogram and the human functional state]. Rostov-on-Don, Rostov State University Publ., 1998. 264 p.

36. Kropotov Yu. D. Kolichestvennaya EEG, kognitivnye vyzvannye potentsialy mozga cheloveka i neiroterapiya [Quantitative EEG, cognitive evoked potentials of the human brain and neurotherapy]. Donetsk, Zaslavskii A. Yu. Publ., 2010. $512 \mathrm{p}$.

37. Knyazev G. G. EEG delta oscillations as a correlate of basic homeostatic and motivational processes. Neuroscience \& Biobehavioral Reviews, 2012, V. 36, Issue 1, pp. 677-695. DOI: 10.1016/j.neubiorev.2011.10.002

38. HarmonyT.The functional significance of delta oscillations in cognitive processing. Frontiers in Integrative Neuroscience, 2013, V.7.DOI: 10.3389/fnint.2013.00083

39. Aftanas L. I., Reva N. V., Varlamov A. A., Pavlov S. V., Makhiev V. P. Analysis of evoked synchronization and desynchronization of EEG at emotional activation in humans: Temporal and topographic characteristics. Zhurnal Vysshei Nervnoi Deyatel'nosti - Journal of Higher Nervous Activity, 2003, V. 53, no. 4, pp. 485-494 (in Russian).

40. Razumnikova O. M., Wolf N. V., Tarasova I. V. Motivation impact on the frequency-spatial characteristics of brain cortex during performing creative 
imaginary and verbal tasks. Zhurnal Vysshei Nervnoi Deyatel'nosti - Journal of Higher Nervous Activity, 2008, V. 58, no. 5, pp. 540-553 (in Russian).

41. Danilova N. N., Strabykina E. A. Frequency-selective generators of oscillatory brain activity allow identifying processes of a working memory. International Journal of Psychophysiology, 2010, V. 77, Issue 3, p. 208. DOI: 10.1016/j.ijpsycho.2010.06.011

42. Danilova N. N., Ushakova T. N., Volkov G. V., Pligina A. M., Strabykina E. A. Displaying semantic categories in brain electrical activity. Eksperimental'naya psikhologiya - Experimental Psychology, 2013, V.6, no. 4, pp. 5-21 (in Russian).

43. Posner M. I., Petersen S. E. The attention system of the human brain. Annual Review of Neuroscience, 1990, V. 13, pp. 25-42.

44. Fan J., McCandliss B. D., Sommer T., Raz A., Posner M. I. Testing the efficiency and independence of attentional networks. Journal of Cognitive Neuroscience, 2002, V. 14, Issue 3, pp. 340-347. DOI: $10.1162 / 089892902317361886$

45. Mihov K. M., Denzler M., Förster J. Hemispheric specialization and creative thinking: a meta-analytic review of lateralization of creativity. Brain and Cognition, 2010, V. 72, no. 3, pp. 442-448.

46. Luriya A. R. Osnovy neiropsikhologii [Fundamentals of neuropsychology]. Moscow, Akademiya Publ., 2003. 384 p.

47. Dumenko V. N., Kozlov M. K. Dynamics of the gamma-band power of induced EEG responses to facial stimuli with increased visual working memory load. Zhurnal Vysshei Nervnoi Deyatel'nosti - Journal of Higher Nervous Activity, 2012, 62 (1), pp. 20-32 (in Russian).

48. Halpern D. F. Sex differences in cognitive abilities. 3rd ed. Mahwah; N.Y.; London: Lawrence Erlbaum Ass. Publ., 2000. 420 p.

49. Thomsen T., Hugdahl K., Ersland L. et al. Functional magnetic resonance imaging (fMRI) study of sex differences in a mental rotation task. Medical Science Monitor, 2000, 6 (6), pp. 1186-1196.

50. Fink A., Neubauer A. C. EEG alpha oscillations during the performance of verbal creativity tasks: Differential effects of sex and verbal intelligence. International Journal of Psychophysiology, 2006, V. 62, Issue 1, pp. 46-53. DOI: $10.1016 /$ j.ijpsycho.2006.01.001

51. Vol'f N. V., Razumnikova O. M., Bryzgalov A. O., Onishchenko M. A., Lapina E. Yu. Neurophysiological analysis of sexual dimorphism mechanisms of cognitive strategies: Effects of selective processes. Byulleten'Sibirskogo otdeleniya Rossiyskoy akademii meditsinskikh nauk - Bulletin of Siberian Branch of Russian Academy of Medical Sciences, 2010, V. 30, no. 4, pp. 6-13 (in Russian).

52. Klimesch W. EEG alpha and theta oscillations reflect cognitive and memory performance: a review and analysis. Brain Research Reviews, 1999, V. 29, Issues 2-3, pp. 169-195. DOI: 10.1016/S0165-0173(98)00056-3 